Review Article

\title{
Research Progress on Chemical Constituents and Anticancer Pharmacological Activities of Euphorbia lunulata Bunge
}

\author{
Yuwei Wang $\mathbb{D},{ }^{1}$ Xiao $Y u^{2}$ Lingna Wang $\mathbb{D},{ }^{1}$ Fang Zhang $\mathbb{D},{ }^{1}$ and Yongqing Zhang $\mathbb{D}^{1}$ \\ ${ }^{1}$ College of Pharmacy, Shandong University of Traditional Chinese Medicine, Jinan 250355, China \\ ${ }^{2}$ Shandong Medicine Technician College, Tai'an 271016, China \\ Correspondence should be addressed to Fang Zhang; zfang_819@163.com and Yongqing Zhang; zyq622003@126.com
}

Received 25 August 2019; Revised 22 November 2019; Accepted 10 December 2019; Published 14 January 2020

Academic Editor: Yunfeng Zhao

Copyright (c) 2020 Yuwei Wang et al. This is an open access article distributed under the Creative Commons Attribution License, which permits unrestricted use, distribution, and reproduction in any medium, provided the original work is properly cited.

Euphorbia lunulata Bunge (ELB) is a traditional Chinese medicine possessing the functions of expectoration, cough relief, asthma relief, detoxification, and itching relief. Modern pharmacological studies have showed that ELB exhibits a variety of activities, such as antitumor, antibacterial, and antioxidant activities. In particular, the anticancer activities of ELB have attracted much attention. In this review, we summarize the recent research progress on the chemical constituents and anticancer activities of ELB by searching the PubMed, Web of Science, and China National Knowledge Infrastructure databases. The results show that more than 151 components have been identified from extracts of ELB, including 73 terpenoids, 28 flavonoids, 8 phenylpropanoids, 7 steroids, 19 phenols, and 5 alkaloids. ELB has been shown to exhibit significant inhibitory effects on lung, cervical, gastric, breast, and liver cancers, and its anticancer effects are mainly manifested in the 3 aspects, including cell cycle arrest, cell apoptosis, and inhibition of the migration of cancer cells.

\section{Introduction}

Cancer, one of the most common malignancy tumors, is a group of diseases characterized by irregular unlimited growth and proliferation of abnormal cells, as well as imbalance of apoptosis [1]. In 2012, the WHO's GLOBOCAN estimated approximately 14.1 million new cancer cases and 8.2 million deaths worldwide, which are expected to rise to 22 million in the next two decades [2]. Current methods for treating cancers, such as chemotherapy, radiotherapy, molecular targeted therapy, and immunotherapy, are not always satisfactory. Among them, molecular targeted therapy and radiotherapy have significant side effects on patients, especially elderly patients. Furthermore, chemotherapy drugs typically have a single selectivity and low tolerance [3-5]. Therefore, finding and developing more effective and safer methods for treating cancers have become an important research direction for researchers.

Research into the sources of new drugs indicates that natural products play an important role in drug discovery and development for the treatment of some human diseases, including for tumors. According to incomplete statistics, anticancer agents derived from botanical drugs accounted for $32.25 \%$ of total anticancer drugs $[6,7]$. In addition, some ingredients extracted from natural products (e.g., camptothecin, taxol, vinblastine, and vincristine) have become the first choice for treating some tumors [8-11]. China is abundant in traditional Chinese medicine (TCM) resources. Chinese herbal medicines have the advantages of rich active ingredients, diverse structures, limited side effects, and a wide anticancer spectrum; therefore, the extraction of new antitumor active compounds from TCM has become a widely popular topic in recent years [12-18].

Euphorbia lunulata Bunge (ELB), recorded as Euphorbia esula L. in Chinese flora [19], is a traditional Chinese medicine, mainly distributed in the northeastern and northern regions of China, which is widely distributed in Eurasia and naturalized in North America and worldwide [19]. ELB possessed the functions of expectoration, cough relief, asthma relief, detoxification, and itching relief and has been widely used in the treatment of phlegm, coughs, asthma, edema, scabies, and anonymous swelling [20], as 
well as for lung cancer, gastric cancer, lymph node nucleus [21], rheumatic immune diseases [22], and psoriasis [23]. Modern pharmacological studies have shown that ELB exhibits a variety of activities, such as antitumor, antibacterial [24], and antioxidant [25] activities. Studies have shown that ELB has significant anticancer activity, whether used alone [26] or in combination with other drugs [27].

In recent years, scholars, both at home and abroad, have showed great interest in ELB because of its anticancer efficacy. Thus, many of the chemical constituents of ELB have been isolated, such as terpenoids, flavonoids, steroids, phenylpropanoids, phenols, and other chemical constituents [28-53]; yet, few of these ingredients have been tested for their anticancer activities. Until recently, not much attention has been paid to the screening of its active anticancer components. Therefore, a comprehensive and systemic review of the chemical constituents of ELB and their associated anticancer activities is indispensable for its further research and clinical applications.

\section{Constituents}

More than 151 compounds have been isolated and identified from ELB so far, including diterpenoids, triterpenes, flavonoids, steroids, alkaloids, coumarins, lignins, phenols, and volatile oils.

2.1. Terpenoids. The terpenoids have been given more attention since the discovery of taxol, and the terpenoids are the most abundant components in ELB. Up to now, 73 terpenoids have been isolated and identified from ELB, which contained 56 diterpenoids and 17 triterpenoids.

2.1.1. Diterpenoids. Euphorbia is rich in diterpenoids. So far, there have been more than 56 kinds of diterpenoids isolated from ELB, which can be classified into four classes: ingenane-type, jatrophane-type, abietane-type, and other types of diterpenes.

Among them, 20 kinds of ingenane-type diterpenoids (see Figure 1) have been isolated from ELB, including 17-benzoyloxy-3-O-(2,3-dimethylbutanoyl)-20-deoxyingenol (1) [28], 17-benzoyloxy-3-O-(2,3-dimethylbutanoyl)-13-(2,3dimethylbutanoyloxy)-20-deoxyingenol (2) [28], 7-benzoyloxy-3-O-(2,3-dimethylbutanoyl)-13-(2,3-dimethylbutanoyloxy)ingenol (3) [28], 7-benzoyloxy-20-O-(2,3dimethylbutanoyl)-13-(2,3-dimethylbutanoyloxy)ingenol (4) [28], 17-benzoyloxy-13-octanoyloxyingenol (5) [28], 3O-benzoyl-17-benzoyloxy-13-octanoyloxyingenol (6) [28], 20-O-benzoyl-17-benzoyloxy-13-octanoyloxyingenol [28], 3-O-benzoyl-17-benzoyloxy-13-octanoyloxy-20-deoxyingenol (8) [28], 17-benzoyloxy-3-O-(2,3-dimethylbutanoyl)-13-octanoyloxyingenol (9) [28], 17-benzoyloxy-20O-(2,3-dimethylbutanoyl)-13-octanoyloxyingenol

(10) [28], 3-O-benzoyl-13,17-dibenzoyloxyingenol (11) [28], 13,17-dibenzoyloxy-3-O-(2,3-dimethylbutanoyl)ingenol (12) [28], 3-O-benzoyl-17-benzoyloxy-13-(2,3-dimethylbutanoyloxy)ingenol (13) [28], 13,17-dibenzoyloxy-3O-(2,3-dimethylbutanoyl)-20-deoxyingenol (14) [28], 3-O- benzoyl-13-octanoyloxyingenol (15) [28], 3-O-(2,3-dimethylbutanoyl)-13-octanoyloxyingenol (16) [28], 6-benzoyloxy-20-deoxyingenol-5-benzoate (17) [29], ingenol-3,20dibenzoate (18) [30], 13,16-dibenzoyloxy-20-deoxyingeno3-benzoate (19) [31], and kansuiphorin D (20) [32].

As shown in Figure 2, 28 kinds of jatrophane-type diterpenoids have been isolated from ELB, such as $2 \alpha, 3 \beta, 5 \alpha, 9 \alpha, 15 \beta$-pentaacetoxy-11,12-epoxy-7 $\beta$-isobutyryl- $8 \alpha$ benzoyloxyjatropha-6(17)-en-14-one (21) [33], salicinolide (22) [34], $2 \alpha, 3 \beta, 5 \alpha, 9 \alpha, 15 \beta$-pentaacetoxy-11,12-epoxy- $7 \beta, 8 \alpha$ diisobutyryloxyjatropha-6(17)-en-14-one (23) [34], $3 \beta, 5 \alpha, 15 \beta$-triacetoxy- $7 \beta$-isobutyryloxy- $9 \alpha$-nicotinoyloxyjatropha-6(17),11(E)-dien-14-one (24) [34], euphoscopin M (25) [32], alisol A (26) [32], euphornin A (27) [32], 14 $\alpha, 15 \beta$ diacetoxy- $3 \alpha, 7 \beta$-dibenzoyloxy-9-oxo- $2 \beta \mathrm{H}, 13-\alpha \mathrm{Hjatropha-}$ 5E,11E-diene (28) [35], euphornin (29) [35], euphoscopin B (30) [35], euphornin $\mathrm{N}$ (31) [35], esulatins $\mathrm{H}(2 \mathrm{R}, 3 \beta, 5 \mathrm{R}$, 8R,9R,12R,15 $\beta$-heptaacetoxy-11,14-epoxy-14R-hydroxy-7 $\beta$ isobutanoyloxyjatropha-6(17)-ene) (32) [36], esulatinsI (3,5,7,15-tetraacetoxy-2-benzoyloxyjatropha-6(17),11-diene-9,14-dione) (33) [36], esulatins $(3 \beta, 5 R, 15 \beta$-triacetoxy- $7 \beta$ isobutanoyloxyjatropha-6(17),11Ediene-9,14-dione) (34) [36], esulatinsK (35) [36], esulatinsL $(2 R, 3 \beta, 5 R, 15 \beta$-tetraacetoxy$7 \beta$-isobutanoyloxy-9R-nicotinoyloxyjatropha-6(17),11E-dien14-one) (36) [36], esulatins $(3 \beta, 5 R, 15 \beta$-triacetoxy- $7 \beta$-isobutanoyloxy-9R-nicotinoyloxyjatropha-6(17),11-dien-14-one) (37) $[36], 2 \mathrm{R}, 3 \beta, 5 \mathrm{R}, 7 \beta, 15 \beta$-pentaacetoxy-9R-nicotinoyloxyjatropha-6(17),11-dien-14-one (38) [36], salicinolide (39) [36], euphosalicin (40) [36], esulatinsA(2R,3R,4S,5R,7S,8R, 9S,11R,12S,13R,15R)-2,3,5,8,9,15hexaacetoxy-11,12-epoxy7-(isobutanoyloxy)jatroph-6(17)en-14-one (41) [37], esulatinsB $(2,3,5,7,15$-pentaacetoxyjatropha-6(17),11-diene-9,14dione) (42) [37], esulatinsC (43) [37], esulatinsD (44) [38], esulatins (2,3,5,15-tetraacetoxyjatropha-6(17),7E,11E-triene9,14-dione) (45) [38], 11,14-epoxy- $3 \beta, 5 \alpha, 7 \beta, 8 \alpha, 9 \alpha, 15 \beta$-hexaacetoxy-12-oxo-13 $\alpha \mathrm{H}$-jatropha-6(17)-ene (46) [39], 1 $\alpha, 3 \beta$ diacetoxy- $5 \alpha, 7 \beta$-dibenzoyloxy-9,14-dioxo- $11 \beta, 12 \alpha$-expoxy$2 \alpha, 8 \alpha, 15 \beta$-trihydroxy-13 $\alpha \mathrm{H}$-jatropha-6(17)-ene (47) [39], and esulol A (48) [40].

5 kinds of abietane-type diterpenoids, including 17hxdroxyjolkinolide A (49) [41], jolkinolide A (50) [41], jolkinolide B (51) [41], 18-hydroxyhelioscopinolide A (52) [32], and Ent-3 $\alpha$-formylabieta-8(14),13(15)-dien-16,12 $\beta$ olide (53) [35], have been isolated from ELB; in addition, 3 other types of diterpenes including esulating (54) [42], 5,8,14-triacetoxy-3-benzoyloxy-15-hydroxy-9-oxo-paraliane (55) [42], and cassipourol (56) [34] have been isolated; the structures of these 8 compounds are shown in Figure 3.

2.1.2. Triterpenes. So far, 17 triterpenoids were isolated from the ELB, which could be classified into tetracyclic triterpenes and pentacyclic triterpenes. Among them, except for euphor (57) [34], the other 10 tetracyclic triterpenes (Figure 4) were all cycloartane-type triterpenes, including 24-methylenecycloartan-3 $\beta$-ol (58) [43], 25-hydroperoxycycloart-23en-3 $\beta$-ol (59) [34], 25-hydroperoxycycloart-23-en-3 $\beta$-ol (60) [34], (23E)-25-methoxycycloart-23-en-3 $\beta$-ol (61) [34], (23E)-cycloart-23,25-dien-3 $\beta$-ol (62) [34], 24- 


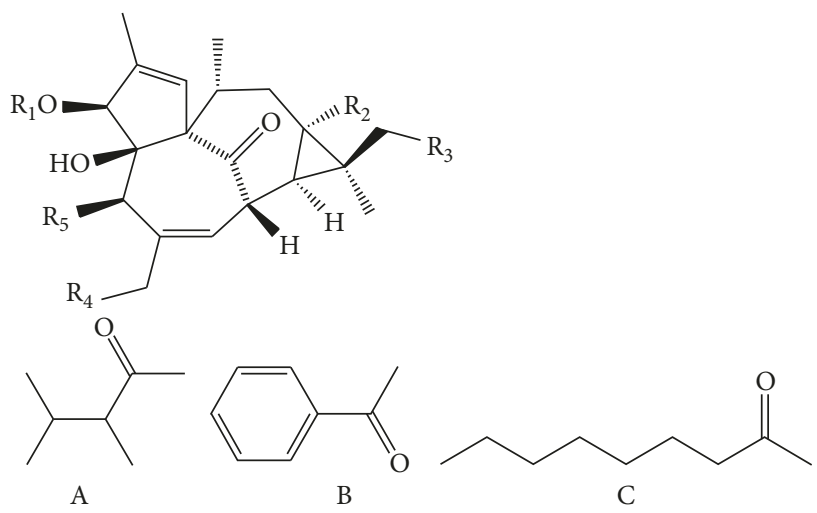

(1) $\mathrm{R}_{1}=\mathrm{A}, \mathrm{R}_{2}=\mathrm{H}, \mathrm{R}_{3}=\mathrm{OB}, \mathrm{R}_{4}=\mathrm{H}, \mathrm{R}_{5}=\mathrm{OH}$

(2) $R_{1}=A, R_{2}=O A, R_{3}=O B, R_{4}=H, R_{5}=O H$

(3) $\mathrm{R}_{1}=\mathrm{A}, \mathrm{R}_{2}=\mathrm{OA}, \mathrm{R}_{3}=\mathrm{OB}, \mathrm{R}_{4}=\mathrm{OH}, \mathrm{R}_{5}=\mathrm{OH}$

(4) $\mathrm{R}_{1}=\mathrm{H}, \mathrm{R}_{2}=\mathrm{OA}, \mathrm{R}_{3}=\mathrm{OB}, \mathrm{R}_{4}=\mathrm{OA}, \mathrm{R}_{5}=\mathrm{OH}$

(5) $\mathrm{R}_{1}=\mathrm{H}, \mathrm{R}_{2}=\mathrm{OC}, \mathrm{R}_{3}=\mathrm{OB}, \mathrm{R}_{4}=\mathrm{OH}, \mathrm{R}_{5}=\mathrm{OH}$

(6) $\mathrm{R}_{1}=\mathrm{B}, \mathrm{R}_{2}=\mathrm{OC}, \mathrm{R}_{3}=\mathrm{OB}, \mathrm{R}_{4}=\mathrm{OH}, \mathrm{R}_{5}=\mathrm{OH}$

(7) $\mathrm{R}_{1}=\mathrm{H}, \mathrm{R}_{2}=\mathrm{OC}, \mathrm{R}_{3}=\mathrm{OB}, \mathrm{R}_{4}=\mathrm{OB}, \mathrm{R}_{5}=\mathrm{OH}$

(8) $\mathrm{R}_{1}=\mathrm{B}, \mathrm{R}_{2}=\mathrm{OC}, \mathrm{R}_{3}=\mathrm{OB}, \mathrm{R}_{4}=\mathrm{H}, \mathrm{R}_{5}=\mathrm{OH}$

(9) $\mathrm{R}_{1}=\mathrm{A}, \mathrm{R}_{2}=\mathrm{OC}, \mathrm{R}_{3}=\mathrm{OB}, \mathrm{R}_{4}=\mathrm{OH}, \mathrm{R}_{5}=\mathrm{OH}$

(10) $\mathrm{R}_{1}=\mathrm{H}, \mathrm{R}_{2}=\mathrm{OC}, \mathrm{R}_{3}=\mathrm{OB}, \mathrm{R}_{4}=\mathrm{OA}, \mathrm{R}_{5}=\mathrm{OH}$

(11) $\mathrm{R}_{1}=\mathrm{B}, \mathrm{R}_{2}=\mathrm{OB}, \mathrm{R}_{3}=\mathrm{OB}, \mathrm{R}_{4}=\mathrm{OH}, \mathrm{R}_{5}=\mathrm{OH}$

(12) $\mathrm{R}_{1}=\mathrm{A}, \mathrm{R}_{2}=\mathrm{OB}, \mathrm{R}_{3}=\mathrm{OB}, \mathrm{R}_{4}=\mathrm{OH}, \mathrm{R}_{5}=\mathrm{OH}$

(13) $\mathrm{R}_{1}=\mathrm{B}, \mathrm{R}_{2}=\mathrm{OA}, \mathrm{R}_{3}=\mathrm{OB}, \mathrm{R}_{4}=\mathrm{OH}, \mathrm{R}_{5}=\mathrm{OH}$

(14) $\mathrm{R}_{1}=\mathrm{A}, \mathrm{R}_{2}=\mathrm{OB}, \mathrm{R}_{3}=\mathrm{OB}, \mathrm{R}_{4}=\mathrm{H}, \mathrm{R}_{5}=\mathrm{OH}$

(15) $\mathrm{R}_{1}=\mathrm{B}, \mathrm{R}_{2}=\mathrm{OC}, \mathrm{R}_{3}=\mathrm{H}, \mathrm{R}_{4}=\mathrm{OH}, \mathrm{R}_{5}=\mathrm{OH}$

(16) $\mathrm{R}_{1}=\mathrm{A}, \mathrm{R}_{2}=\mathrm{OC}, \mathrm{R}_{3}=\mathrm{H}, \mathrm{R}_{4}=\mathrm{OH}, \mathrm{R}_{5}=\mathrm{OH}$

(17) $\mathrm{R}_{1}=\mathrm{H}, \mathrm{R}_{2}=\mathrm{H}, \mathrm{R}_{3}=\mathrm{OB}, \mathrm{R}_{4}=\mathrm{H}, \mathrm{R}_{5}=\mathrm{OB}$

(18) $\mathrm{R}_{1}=\mathrm{B}, \mathrm{R}_{2}=\mathrm{H}, \mathrm{R}_{3}=\mathrm{H}, \mathrm{R}_{4}=\mathrm{OB}, \mathrm{R}_{5}=\mathrm{OH}$

(19) $\mathrm{R}_{1}=\mathrm{B}, \mathrm{R}_{2}=\mathrm{OB}, \mathrm{R}_{3}=\mathrm{OB}, \mathrm{R}_{4}=\mathrm{H}, \mathrm{R}_{5}=\mathrm{OH}$

(20) $\mathrm{R}_{1}=\mathrm{B}, \mathrm{R}_{2}=\mathrm{H}, \mathrm{R}_{3}=\mathrm{H}, \mathrm{R}_{4}=\mathrm{H}, \mathrm{R}_{5}=\mathrm{COCH}_{3}$

FIgURE 1: The structures of 20 ingenane-type diterpenoids (1-20).

methylenecycloartan-3 $\beta, 28$-diol (63) [34], 3-hydroxylcycloartane-25-ene (64) [34], 25,26,27-trinor-3 $\alpha$ hydroxycycloartan-24-oic acid (65) [35], cycloart-23ene-3 $\beta, 25$-diol (66) [35], and cycloart-23-ene- $3 \beta, 25,28$ triol (67) [35]. And 6 pentacyclic triterpenes including three oleanane-type triterpenoids (68-70), one ursanetype triterpenoid (71), and two lupane-type triterpenoids $(\mathbf{7 2}, \mathbf{7 3})$, respectively, named $\beta$-amyrin (68) [43], corosolic acid (69) [45], ursolic acid (70) [35], uvaol (71) [34], lupeol (72) [34], and betulin (73) [34], have been isolated and identified. And the structure of 6 pentacyclic triterpenes is presented in Figure 5.

2.2. Flavonoids. Until now, more than 28 flavonoids have been isolated from ELB, which could be structurally divided into dihydroflavones, isoflavones, and flavonoids. Among them, 26 flavonoids (74-99), such as 3,5,7-trihydroxy-8methoxyflavone (74) [43], naringenin-7-O- $\beta$-D-glucoside (75) [22], quercetin-3-O-(6" -galloyl)- $\beta$-D-galactopyranoside (76) [22], apigenin-7-O- $\beta$-D-glucoside (77) [22],
kaempferol-7-O- $\beta$-D-glucoside (78) [44], quercetin-7-O$\beta$-D-glucoside (79) [44], quercetin-3-O- $\alpha$-L-rha (80) [44], kaempferol-3-O-(6"-galloyl)- $\beta$-D-glucoside (81) [44], kaempferol-3-O- $\beta$-D-glucoside (82) [44], jaceosidin (83) [45], myricetin-3-O-(2",3" -digalloyl)- $\beta$-D-galactopyranoside (84) [46], myricetin-3-O-( $2^{\prime \prime}$-galloyl)- $\beta$-D-galactopyranoside (85) [46], myricitrin (86) [46], myricetin (87) [46], apigenin (88) [46], luteolin (89) [46], 3-O-methylquercetin (90) [46], 5,7,2',5'-tetrahydroxyflavone (91) [46], kaempferol (92) [47], quercetin (93) [47], quercetin-3-L-rha (94) [47], kaempferol-3-L-rha (95) [47], quercetin-3-O- $\left(2^{\prime \prime}, 3^{\prime \prime}\right.$ digalloyl)- $\beta$-D-galactopyranoside (96) [48], quercetin-3-O( $2^{\prime \prime}$-galloyl)- $\beta$-D-galactopyranoside (97) [48], hyperin (98) [48], and quercetin-3-0- $\beta$-glucuronic (99) [49], have been isolated and identified from ELB. In addition, a dihydroflavone and an isoflavone, named 5,7,4'-trihydroxydihydroflavone (100) [45] and isoquercetin (101) [46], was isolated and identified. The structures of these 28 flavonoids are presented in Table 1 and Figure 6.

2.3. Phenylpropanoid. 8 phenylpropanoids (102-109) were isolated from ELB, which could be divided into two classes: coumarins and lignans. 5 coumarins have been isolated, including scopolin (102) [41], scopoletin (103) [22], isofraxidin (104) [22], esculetin (105) [47], and maoyancaosu (106) $[47,50] .3$ lignans were isolated and identified from ELB, respectively, named trans-2-(4" -hydroxy-3" -methoxybenzyl)-3-(3', $4^{\prime}$-dimethoxybenzyl)-butyolactone (107) [51], trans-2-(3", $4^{\prime \prime}$-dimethoxybenzyl)-3-( $3^{\prime}, 4^{\prime}$-dimethoxybenzyl)-butyolactone (108) [51], and isoanmericanon A (109) [51]. The structures of 8 phenylpropanoids are listed in Figure 7.

2.4. Steroids. As shown in Figure 8,7 steroids have been isolated from ELB, including $\beta$-sitosterol (110) [44], stigmasterol (111) [44], 3 $\beta$-hydroxy-7 $\beta$-methoxy-5-stigmasterone (112) $[45], 3 \beta, 22 \beta$-dihydroxy-7 $\beta$-methoxy-5-stigmasterone (113) [45], $\beta$-daucosterol (114) [32], stigmast-4-ene-3 $\beta, 6 \beta$ diol (115) [35], and stigmastane-3,6-dione (116) [35].

2.5. Phenols. 19 phenols (117-135) have been obtained from ELB, including 1,3,4,6-tetra-O-galloyl- $\beta$-D-glucose (117) [46], 1,2,6-tri-O-galloyl- $\beta$-D-glucose (118) [46], 1,3,6-tri-Ogalloyl- $\beta$-D-glucose (119) [46], 3,3'-di-O-methylellagic acid (120) [46], ellagic acid (121) [46], gallic acid (122) [46], protocatechuic acid (123) [46], 3,4,5-trimethoxybenzoic acid (124) [46], 2,6-dihydroxyacetophenone (125) [46], ethyl gallate (126) [51], octadecyl caffeate (127) [41], hexadecyl ferulate (128) [41], p-hydroxybenzoic acid (129) [45], 3hydroxy-4-methoxybenzoic acid (130) [45], 2,5-dihydroxy4-methoxy phenanthrene (131) [45], erigeside C (132) [45], 4,8-dihydroxy-1-tetralone (133) [52], methyl 3,4-dihydroxybenzoate (134) [52], and vifolin (135) [43]. Their structures are listed in Figure 9.

2.6. Alkaloids. A few of alkaloids were found from ELB, including asperglaucide (136) [45], 1H-3-amido oxime (137) 


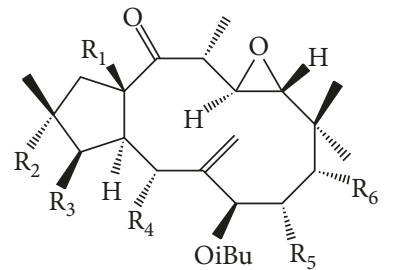

(21) $\mathrm{R}_{1}=\mathrm{R}_{2}=\mathrm{R}_{3}=\mathrm{R}_{4}=\mathrm{R}_{6}=\mathrm{OiBu}, \mathrm{R}_{5}=\mathrm{OBz}$

(22) $\mathrm{R}_{1}=\mathrm{R}_{2}=\mathrm{R}_{3}=\mathrm{R}_{4}=\mathrm{R}_{6}=\mathrm{OAc}, \mathrm{R}_{5}=\mathrm{OiBu}$

(44) $\mathrm{R}_{1}=\mathrm{R}_{2}=\mathrm{R}_{3}=\mathrm{R}_{4}=\mathrm{R}_{5}=\mathrm{R}_{6}=\mathrm{OAc}$

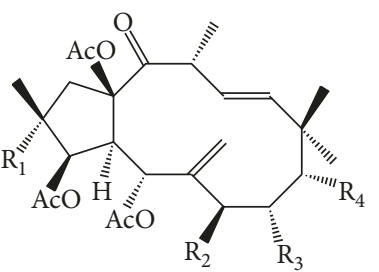

(24) $\mathrm{R}_{1}=\mathrm{H}, \mathrm{R}_{2}=\mathrm{OiBu}, \mathrm{R}_{3}=\mathrm{H}, \mathrm{R}_{4}=\mathrm{ONic}$

(35) $\mathrm{R}_{1}=$ ONic, $\mathrm{R}_{2}=\mathrm{OAc}, \mathrm{R}_{3}=\mathrm{H}, \mathrm{R}_{4}=\mathrm{ONic}$

(36) $\mathrm{R}_{1}=\mathrm{OAc}, \mathrm{R}_{2}=\mathrm{OiBu}, \mathrm{R}_{3}=\mathrm{H}, \mathrm{R}_{4}=\mathrm{ONic}$

(37) $\mathrm{R}_{1}=\mathrm{H}, \mathrm{R}_{2}=\mathrm{OiBu}, \mathrm{R}_{3}=\mathrm{H}, \mathrm{R}_{4}=\mathrm{ONic}$

(38) $\mathrm{R}_{1}=\mathrm{OAc}, \mathrm{R}_{2}=\mathrm{OAc}, \mathrm{R}_{3}=\mathrm{H}, \mathrm{R}_{4}=\mathrm{ONic}$

(42) $\mathrm{R}_{1}=\mathrm{OAc}, \mathrm{R}_{2}=\mathrm{OAc}, \mathrm{R}_{3}=\mathrm{H}, \mathrm{R}_{4}=\mathrm{OAc}$

(43) $\mathrm{R}_{1}=\mathrm{OAc}, \mathrm{R}_{2}=\mathrm{OiBu}, \mathrm{R}_{3}=\mathrm{OAc}, \mathrm{R}_{4}=\mathrm{OAc}$

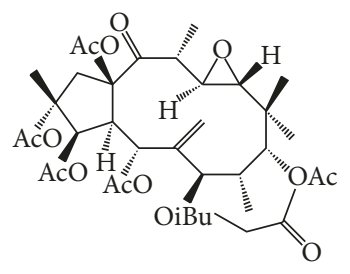

(23)

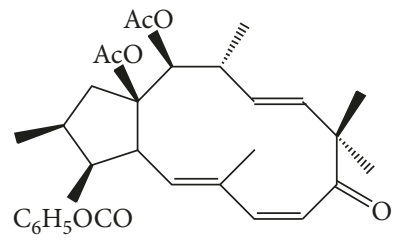

(25)

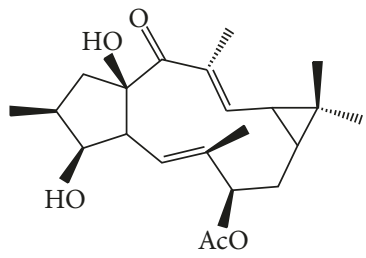

(26)

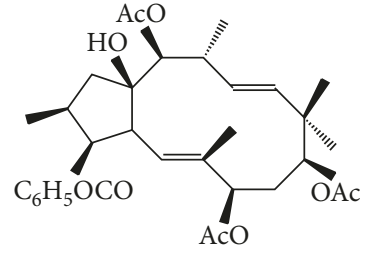

(27)
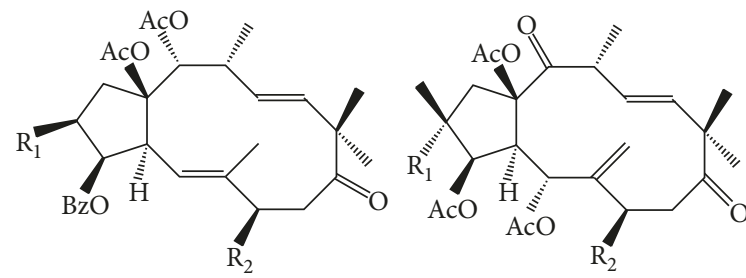
(28) $\mathrm{R}_{1}=\mathrm{CH}_{3}, \mathrm{R}_{2}=\mathrm{OBz}$
(33) $\mathrm{R}_{1}=\mathrm{OBz}, \mathrm{R}_{2}=\mathrm{OAc}$
(30) $\mathrm{R}_{1}=\mathrm{H}, \mathrm{R}_{2}=\mathrm{OAc}$
(34) $\mathrm{R}_{1}=\mathrm{H}, \mathrm{R}_{2}=\mathrm{OiBu}$
(31) $\mathrm{R}_{1}=\mathrm{CH}_{3}, \mathrm{R}_{2}=\mathrm{OAc}$
(41) $\mathrm{R}_{1}=\mathrm{OAc}, \mathrm{R}_{2}=\mathrm{OAc}$

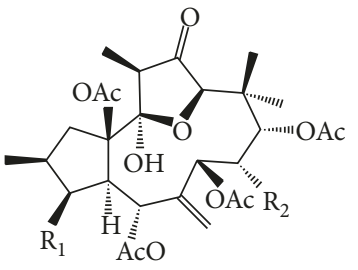

(46) $\mathrm{R}_{1}=\mathrm{OAc}, \mathrm{R}_{2}=\mathrm{OAc}$

(48) $\mathrm{R}_{1}=\mathrm{OH}, \mathrm{R}_{2}=\mathrm{OBz}$

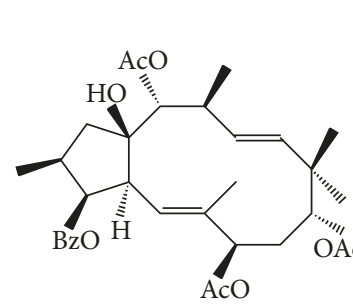

(29)

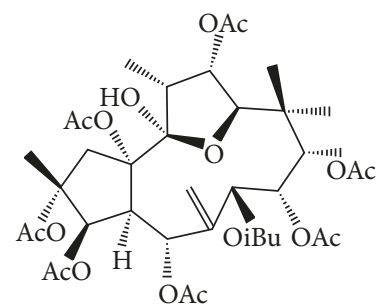

(32)

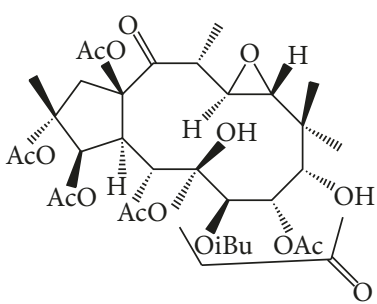

(39)

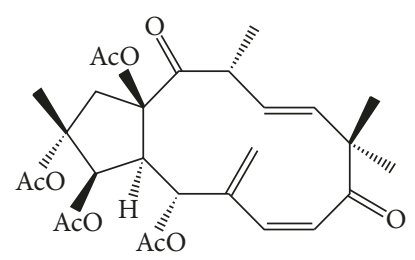

(45)

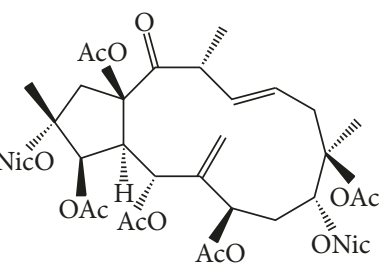

(40)

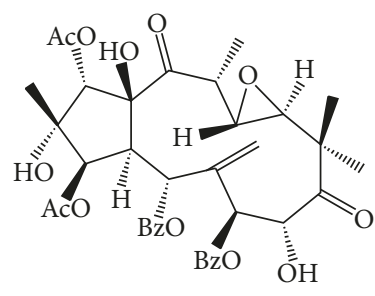

(47)

FIGURE 2: The structures of 28 jatrophane-type diterpenoids (21-48). 
<smiles></smiles>

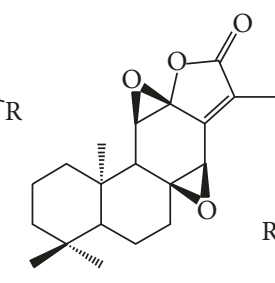

(51)

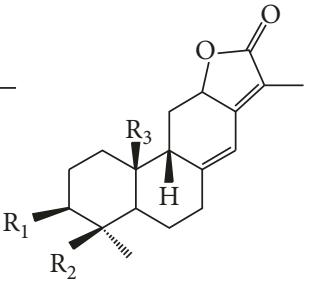

(52) $\mathrm{R}_{1}=\mathrm{OH}, \mathrm{R}_{2}=\mathrm{CH}_{2} \mathrm{OH}, \mathrm{R}_{3}=\mathrm{CH}_{3}$ (53) $\mathrm{R}_{1}=\mathrm{OAc}, \mathrm{R}_{2}=\mathrm{CH}_{3}, \mathrm{R}_{3}=\mathrm{H}$
(49) $\mathrm{R}=\mathrm{CH}_{2} \mathrm{OH}$

(50) $\mathrm{R}=\mathrm{CH}_{3}$

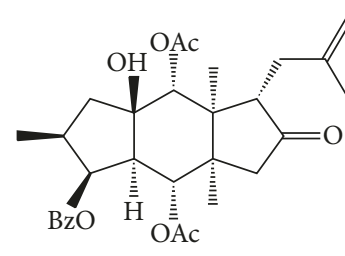

(54)

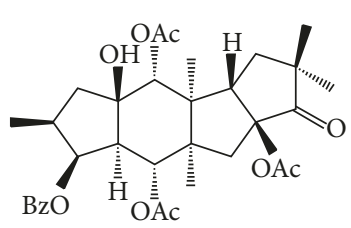

(55)

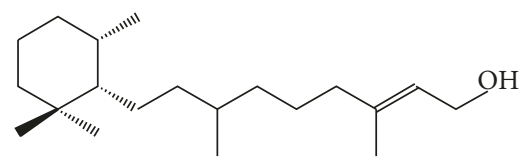

(56)

FIgURE 3: The structures of 5 abietane type and 3 other types of diterpenoids (49-56).

[52], $N$-[2-(1H-indol-3-yl)ethyl] acetamide (138) [52], $N$-[2(4-hydroxyphenyl)ethyl]acetamide (139) [52], and 2,3dihydroxy-methyl nitrogen (140) [52]. Their structures are presented in Figure 10.

2.7. Long-Chain Aliphatic Group. 4 compounds, which belonged to the long-chain aliphatic group, were isolated, including $n$-octadecyl alcohol (141) [41], cetyl alcohol (142) [44], triacontanol (143) [44], and tetracatanol (144) [44].

2.8. Volatile Oils. Wang et al. [53] used steam distillation to extract volatile oil from ELB and analyze the chemical constituents of volatile oil by gas chromatography-mass spectrometry (GC-MS). 43 components were identified, and the relative percentage of each component was calculated by the peak area normalization method. The identified components accounted for $75.86 \%$ of the total outflow peak area. Among them, 3 main compounds including 3,4,4-trimethyl-2-cyclopenten-1-one (12.67\%) (145), phenylacetaldehyde (12.36\%) (146), $\alpha$-terpineol (4.47\%) (147) and 2 active ingredients including eucalyptol (148) and borneol (149) were identified. Their structures are shown in Figure 11.

2.9. Others. Other chemical constituents other than terpenoids, phenylpropanoids, flavonoids, steroids, phenols, alkaloids, long-chain aliphatic group, volatile oils, such as $o$ phthalic acid bis-(2-ethyl decyl)-ester (150) [41], and sucrose<smiles>CCC(C)=CCCC(C)C1CCC2(C)C3CCC4[C@H](C)[C@@H](O)CC[C@]4(C)C3CCC12C</smiles>

(57)

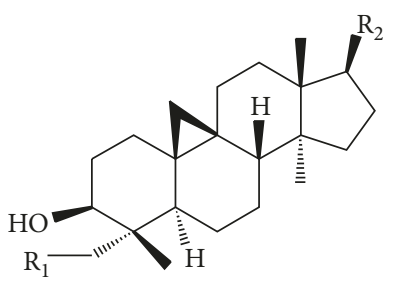<smiles>C=C(C)C=CCC(C)C</smiles>

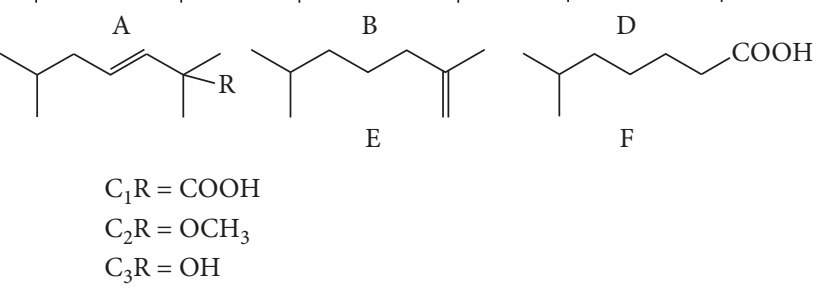

(58) $\mathrm{R}_{1}=\mathrm{H}, \mathrm{R}_{2}=\mathrm{A}$

(59) $\mathrm{R}_{1}=\mathrm{H}, \mathrm{R}_{2}=\mathrm{B}$

(60) $\mathrm{R}_{1}=\mathrm{H}, \mathrm{R}_{2}=\mathrm{C}_{1}$

(61) $\mathrm{R}_{1}=\mathrm{H}, \mathrm{R}_{2}=\mathrm{C}_{2}$

(62) $\mathrm{R}_{1}=\mathrm{H}, \mathrm{R}_{2}=\mathrm{D}$

(63) $\mathrm{R}_{1}=\mathrm{OH}, \mathrm{R}_{2}=\mathrm{A}$

(64) $\mathrm{R}_{1}=\mathrm{H}, \mathrm{R}_{2}=\mathrm{E}$

(65) $\mathrm{R}_{1}=\mathrm{H}, \mathrm{R}_{2}=\mathrm{F}$

(66) $\mathrm{R}_{1}=\mathrm{H}, \mathrm{R}_{2}=\mathrm{C}_{3}$

(67) $\mathrm{R}_{1}=\mathrm{OH}, \mathrm{R}_{2}=\mathrm{C}_{3}$

FIgURE 4: The structures of 11 tetracyclic triterpenoids 57-67.

(151) [41], were isolated, the structures of which are shown in Figure 12.

\section{Antitumor Pharmacological Activity}

At present, the mechanism of the occurrence and development of cancer has not been fully revealed; however, it has been confirmed that the proliferation of cancer cells originates from their ability to avoid programmed cell death (i.e., the so-called apoptosis). Therefore, inducing cancer cell apoptosis has been identified as a target for cancer treatment [54-57]. ELB has exhibited antitumor activity, having significant inhibitory effects on lung, cervical, gastric, breast, and liver cancers. Its anticancer effects are mainly manifested in the following aspects: (1) inhibiting the growth of cancer cells by regulating cell cycles, (2) inducing cancer cell apoptosis by regulating the expression of apoptosis-related proteins, and (3) inhibiting the migration of cancer cells by regulating related signaling pathways. 


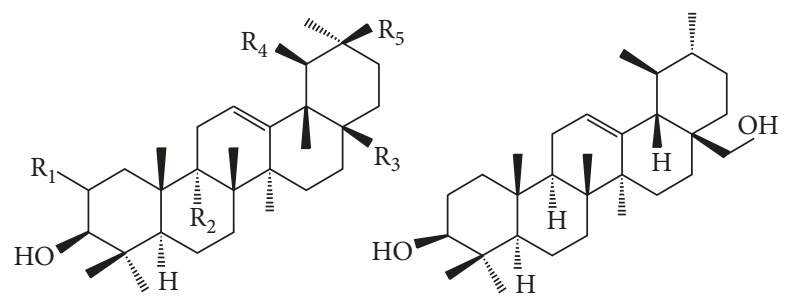

(68) $\mathrm{R}_{1}=\mathrm{R}_{4}=\mathrm{H}, \mathrm{R}_{2}=\mathrm{R}_{3}=\mathrm{R}_{5}=\mathrm{CH}_{3}$

(69) $\mathrm{R}_{1}=\mathrm{OH}, \mathrm{R}_{2}=\mathrm{R}_{5}=\mathrm{H}, \mathrm{R}_{3}=\mathrm{COOH}, \mathrm{R}_{4}=\mathrm{CH}_{3}$

(70) $\mathrm{R}_{1}=\mathrm{R}_{2}=\mathrm{R}_{5}=\mathrm{H}, \mathrm{R}_{3}=\mathrm{COOH}, \mathrm{R}_{4}=\mathrm{CH}_{3}$

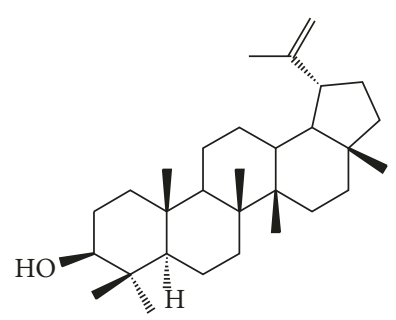

(72)

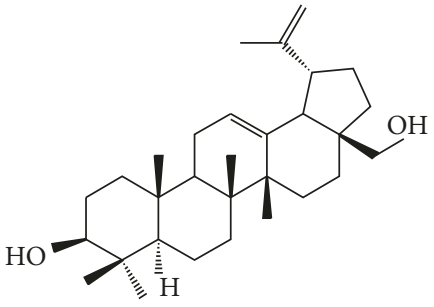

(73)

FIgURE 5: The structures of 6 pentacyclic triterpenoids 68-73.

TABle 1: The structures of 26 flavonoids 74-99.

\begin{tabular}{|c|c|c|c|c|c|c|c|c|}
\hline \multirow{2}{*}{ Comp. number } & \multicolumn{7}{|c|}{ Substitutional groups } & \multirow{2}{*}{ References } \\
\hline & $\mathrm{R}_{1}$ & $\mathrm{R}_{2}$ & $\mathrm{R}_{3}$ & $\mathrm{R}_{4}$ & $\mathrm{R}_{5}$ & $\mathrm{R}_{6}$ & $\mathrm{R}_{7}$ & \\
\hline 74 & $\mathrm{OH}$ & $\mathrm{OH}$ & $\mathrm{OCH}_{3}$ & $\mathrm{H}$ & $\mathrm{H}$ & $\mathrm{H}$ & $\mathrm{H}$ & {$[43]$} \\
\hline 75 & $\mathrm{H}$ & $\mathrm{O}-\beta$-D-Glc & $\mathrm{H}$ & $\mathrm{H}$ & $\mathrm{OH}$ & $\mathrm{OCH}_{3}$ & $\mathrm{H}$ & {$[22]$} \\
\hline 76 & O-(6"-Galloyl)- $\beta$-D-Gal & $\mathrm{OH}$ & $\mathrm{H}$ & $\mathrm{H}$ & $\mathrm{OH}$ & $\mathrm{OH}$ & $\mathrm{H}$ & {$[22]$} \\
\hline 77 & $\mathrm{H}$ & $\mathrm{O}-\beta$-D-Glc & $\mathrm{H}$ & $\mathrm{H}$ & $\mathrm{H}$ & $\mathrm{OH}$ & $\mathrm{H}$ & {$[22]$} \\
\hline 78 & $\mathrm{OH}$ & $\mathrm{O}-\beta$-D-Glc & $\mathrm{H}$ & $\mathrm{H}$ & $\mathrm{H}$ & $\mathrm{OH}$ & $\mathrm{H}$ & {$[44]$} \\
\hline 79 & $\mathrm{OH}$ & O- $\beta$-D-Glc & $\mathrm{H}$ & $\mathrm{H}$ & $\mathrm{OH}$ & $\mathrm{OH}$ & $\mathrm{H}$ & {$[44]$} \\
\hline 80 & O- $\alpha$-L-Rha & $\mathrm{OH}$ & $\mathrm{H}$ & $\mathrm{H}$ & $\mathrm{OH}$ & $\mathrm{OH}$ & $\mathrm{H}$ & {$[44]$} \\
\hline 81 & O-(6"-O-Galloyl)- $\beta-\mathrm{D}-\mathrm{Glc}$ & $\mathrm{OH}$ & $\mathrm{H}$ & $\mathrm{H}$ & $\mathrm{H}$ & $\mathrm{OH}$ & $\mathrm{H}$ & {$[44]$} \\
\hline 82 & O- $\beta$-D-Glc & $\mathrm{OH}$ & $\mathrm{H}$ & $\mathrm{H}$ & $\mathrm{H}$ & $\mathrm{OH}$ & $\mathrm{H}$ & {$[44]$} \\
\hline 83 & $\mathrm{H}$ & $\mathrm{OH}$ & $\mathrm{H}$ & $\mathrm{H}$ & $\mathrm{H}$ & $\mathrm{OCH}_{3}$ & $\mathrm{H}$ & {$[45]$} \\
\hline 84 & $\mathrm{O}-\left(2^{\prime \prime}, 3^{\prime \prime}\right.$-Digalloyl $)-\beta-\mathrm{D}-\mathrm{Gal}$ & $\mathrm{OH}$ & $\mathrm{H}$ & $\mathrm{H}$ & $\mathrm{OH}$ & $\mathrm{OH}$ & $\mathrm{OH}$ & {$[46]$} \\
\hline 85 & O-(2"-Galloyl)- $\beta$-D-Gal & $\mathrm{OH}$ & $\mathrm{H}$ & $\mathrm{H}$ & $\mathrm{OH}$ & $\mathrm{OH}$ & $\mathrm{OH}$ & {$[46]$} \\
\hline 86 & O- $\alpha$-L-Rha & $\mathrm{OH}$ & $\mathrm{H}$ & $\mathrm{H}$ & $\mathrm{OH}$ & $\mathrm{OH}$ & $\mathrm{OH}$ & {$[46]$} \\
\hline 87 & $\mathrm{OH}$ & $\mathrm{OH}$ & $\mathrm{H}$ & $\mathrm{H}$ & $\mathrm{OH}$ & $\mathrm{OH}$ & $\mathrm{OH}$ & [46] \\
\hline 88 & $\mathrm{H}$ & $\mathrm{OH}$ & $\mathrm{H}$ & $\mathrm{H}$ & $\mathrm{H}$ & $\mathrm{OH}$ & $\mathrm{H}$ & {$[46]$} \\
\hline 89 & $\mathrm{H}$ & $\mathrm{OH}$ & $\mathrm{H}$ & $\mathrm{H}$ & $\mathrm{OH}$ & $\mathrm{OH}$ & $\mathrm{H}$ & {$[46]$} \\
\hline 90 & $\mathrm{OCH}_{3}$ & $\mathrm{OH}$ & $\mathrm{H}$ & $\mathrm{H}$ & $\mathrm{OH}$ & $\mathrm{OH}$ & $\mathrm{H}$ & {$[46]$} \\
\hline 91 & $\mathrm{OH}$ & $\mathrm{H}$ & $\mathrm{H}$ & $\mathrm{OH}$ & $\mathrm{H}$ & $\mathrm{H}$ & $\mathrm{OH}$ & {$[46]$} \\
\hline 92 & $\mathrm{OH}$ & $\mathrm{OH}$ & $\mathrm{H}$ & $\mathrm{H}$ & $\mathrm{H}$ & $\mathrm{OH}$ & $\mathrm{H}$ & {$[47]$} \\
\hline 93 & $\mathrm{OH}$ & $\mathrm{OH}$ & $\mathrm{H}$ & $\mathrm{H}$ & $\mathrm{OH}$ & $\mathrm{OH}$ & $\mathrm{H}$ & {$[47]$} \\
\hline 94 & O-L-Rha & $\mathrm{OH}$ & $\mathrm{H}$ & $\mathrm{H}$ & $\mathrm{OH}$ & $\mathrm{OH}$ & $\mathrm{H}$ & {$[47]$} \\
\hline 95 & O-L-Rha & $\mathrm{OH}$ & $\mathrm{H}$ & $\mathrm{H}$ & $\mathrm{H}$ & $\mathrm{OH}$ & $\mathrm{H}$ & [47] \\
\hline 96 & $\mathrm{O}-\left(2^{\prime \prime}, 3^{\prime \prime}\right.$-Digalloyl $)-\beta-\mathrm{D}-\mathrm{Gal}$ & $\mathrm{OH}$ & $\mathrm{H}$ & $\mathrm{H}$ & $\mathrm{OH}$ & $\mathrm{OH}$ & $\mathrm{H}$ & {$[48]$} \\
\hline 97 & O- $\left(2^{\prime \prime}\right.$-Galloyl $)-\beta-D-G a l$ & $\mathrm{OH}$ & $\mathrm{H}$ & $\mathrm{H}$ & $\mathrm{OH}$ & $\mathrm{OH}$ & $\mathrm{H}$ & {$[48]$} \\
\hline 98 & $\mathrm{O}-\beta-\mathrm{D}-\mathrm{Gal}$ & $\mathrm{OH}$ & $\mathrm{H}$ & $\mathrm{H}$ & $\mathrm{OH}$ & $\mathrm{OH}$ & $\mathrm{H}$ & {$[48]$} \\
\hline 99 & $\mathrm{O}-\beta$-D-Glc & $\mathrm{OH}$ & $\mathrm{H}$ & $\mathrm{H}$ & $\mathrm{OH}$ & $\mathrm{OH}$ & $\mathrm{H}$ & [49] \\
\hline
\end{tabular}

3.1. Lung Cancer. Lung cancer is one of the most common malignant tumors, seriously threatening human health. Its incidence and mortality rank high [58].

Xiao et al. and Zhang et al. [59, 60] reported that an aqueous extract of ELB exhibited significant inhibitory activities against the growth of Lewis lung cancer in a mouse model: a flow cytometry assay showed that the $S$ phase percentage of the cell cycle was increased at doses of $30 \mathrm{~g} \cdot \mathrm{kg}^{-1}$ and $60 \mathrm{~g} \cdot \mathrm{kg}^{-1}$ with apoptotic rates of $(16.43 \pm 18.69) \%$ and $(24.37 \pm 15.48) \%$, respectively. The apoptotic index (AI) of the $60 \mathrm{~g} \cdot \mathrm{kg}^{-1}$ group was significantly higher than the control group, with a value of $(5.93 \pm 5.96) \%$. 
<smiles>[R]c1cc(-c2oc3c([R])c([R])cc(O)c3c(=O)c2[R])cc([R])c1[R]</smiles>

(74)-(99)<smiles>O=C1CC(c2ccc(O)cc2)Oc2cc(O)cc(O)c21</smiles>

(100)<smiles>O=c1c(-c2ccc(O)c(O)c2)c(O)oc2cc(O)cc(O)c12</smiles>

(101)
FIgURE 6: The structures of 28 flavonoids 74-101.
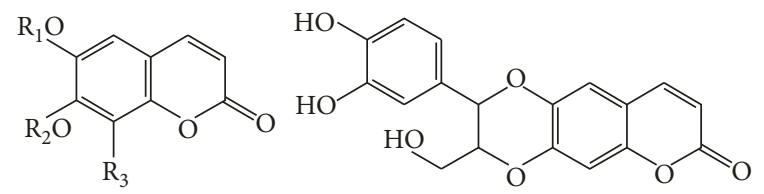

(102) $\mathrm{R}_{1}=\mathrm{CH}_{3}, \mathrm{R}_{2}=\mathrm{Glc}, \mathrm{R}_{3}=\mathrm{H}$

(106)

(103) $\mathrm{R}_{1}=\mathrm{CH}_{3}, \mathrm{R}_{2}=\mathrm{H}, \mathrm{R}_{3}=\mathrm{H}$

(104) $\mathrm{R}_{1}=\mathrm{CH}_{3}, \mathrm{R}_{2}=\mathrm{H}, \mathrm{R}_{3}=\mathrm{OCH}_{3}$

(105) $\mathrm{R}_{1}=\mathrm{R}_{2}=\mathrm{R}_{3}=\mathrm{H}$<smiles>CCOC(=O)C1CC(Cc2ccc3c(c2)OCO3)C1Cc1ccc(O)c(OC)c1</smiles>

(107)<smiles>C=CCC1COC(=O)C1CC1CCC2OCOC2C1</smiles>

(108)

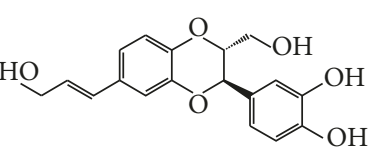

(109)
Figure 7: The structures of 8 phenylpropanoids (102-109).

Therefore, ELB could induce apoptosis at a dose of $60 \mathrm{~g} \cdot \mathrm{kg}^{-1}$. Jiang [61] found that a flavonoid extract of ELB could inhibit the growth of Lewis lung cancer mice and that rabbit serum containing ELB could significantly inhibit the proliferation of A549 lung cancer cells in a concentration- and timedependent manner: at a concentration of $20 \%$ for $72 \mathrm{~h}$, the A549 proliferation inhibiting rate was $39.08 \%$; thus, rabbit serum containing ELB extract could induce significant A549 cell apoptosis by arresting the cell in the G1 phase. Western blotting analysis showed that the active ingredients of ELB could inhibit the phosphorylation of EGFR and downregulate AKT and ERK signals. However, Wang et al. [62] found that extracts of ELB could induce the apoptosis of A549 cells, which may be related to an increase in bax expression and a decrease of bcl-2 expression. Liu et al. [35] evaluated in vitro antiproliferative activities against NCIH460 human lung carcinoma cell lines of five compounds (compounds 28-31 and 53), where the results showed that compound $\mathbf{5 3}$ exhibited marked activity, with an IC50 value
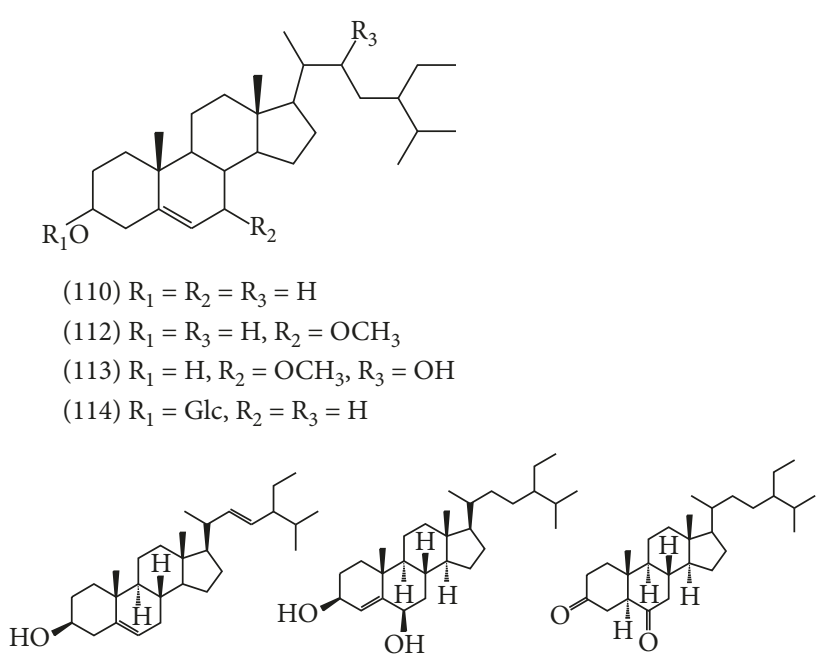

(111)

(115)

(116)

Figure 8: The structures of 7 steroids (110-116).

of $19.5 \mu \mathrm{M}$, and compounds $28-31$ showed moderate cytotoxic activities, with IC50 values of 58.2 (compound 28), 53.1 (compound 29), 33.0 (compound 30), and 36.7 (compound 31) $\mu \mathrm{M}$.

3.2. Cervical Cancer. Cervical cancer is the second most common cancer, affecting women's health worldwide [63]. Ming et al. [64] discovered that an ELB extract could efficiently inhibit HeLa cervical cancer cell proliferation and induce the apoptosis of HeLa cells by downregulating the expression levels of Cyclin D1, Cyclin E, and CDK4 proteins and upregulating the expression levels of the $\mathrm{P} 21$ protein. $\mathrm{Tu}$ et al. [65] reported that an ELB extract could inhibit SiHa human cervical cancer cell proliferation and induce cell apoptosis by increasing the expression levels of caspase-3 and caspase-9.

3.3. Gastric Cancer. Cancer multidrug resistance is the term used to describe a phenomenon in which once a cancer develops resistance to one chemotherapeutic drug, it will become resistant to many (or all) other chemotherapeutic drugs (which may or may not have the same mechanism of action). Cancer multidrug resistance has become a problem that cannot be ignored in clinical practice, and many studies are being carried out to find ways to combat or reverse it [66-70]. Fu et al. reported that the proliferation, migration, and invasion of multidrug-resistant human gastric cancer cell line SGC7901/ADR were significantly inhibited by an $n$ hexane extract of ELB in a time- and dose-dependent manner: the cell cycle was arrested in the G2/M phase, and cell apoptosis was induced. The result of the related mechanisms showed that the extract could upregulate the expression of the apoptosis-promoting protein $\mathrm{Bax}$ and downregulate that of the apoptosis-inhibiting protein $\mathrm{Bcl}-2$, as well as increasing the activities of caspase-3, caspase-8, and caspase-9 [71, 72]. In addition, the ELB extract could increase the sensitivity of the multidrug-resistant human 


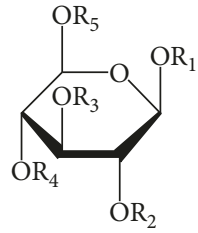

(117) $\mathrm{R}_{1}=\mathrm{R}_{3}=\mathrm{R}_{4}=\mathrm{R}_{5}=\mathrm{G}, \mathrm{R}_{2}=\mathrm{H}$

(118) $\mathrm{R}_{1}=\mathrm{R}_{2}=\mathrm{R}_{5}=\mathrm{G}, \mathrm{R}_{3}=\mathrm{R}_{4}=\mathrm{H}$

(119) $R_{1}=R_{3}=R_{5}=G, R_{2}=R_{4}=H$<smiles>[R]C(=O)c1c([R])c([R])c([R])c([R])c1[R]</smiles>

(122) $\mathrm{R}_{1}=\mathrm{R}_{5}=\mathrm{H}, \mathrm{R}_{2}=\mathrm{R}_{3}=\mathrm{R}_{4}=\mathrm{R}_{6}=\mathrm{OH}$

(123) $\mathrm{R}_{1}=\mathrm{R}_{4}=\mathrm{R}_{5}=\mathrm{H}, \mathrm{R}_{2}=\mathrm{R}_{3}=\mathrm{R}_{6}=\mathrm{OH}$

(124) $\mathrm{R}_{1}=\mathrm{R}_{5}=\mathrm{H}, \mathrm{R}_{2}=\mathrm{R}_{3}=\mathrm{R}_{4}=\mathrm{OCH}_{3}, \mathrm{R}_{6}=\mathrm{OH}$

(125) $\mathrm{R}_{1}=\mathrm{R}_{5}=\mathrm{OH}, \mathrm{R}_{2}=\mathrm{R}_{3}=\mathrm{R}_{4}=\mathrm{H}, \mathrm{R}_{6}=\mathrm{CH}_{3}$

(126) $\mathrm{R}_{1}=\mathrm{R}_{5}=\mathrm{H}, \mathrm{R}_{2}=\mathrm{R}_{3}=\mathrm{R}_{4}=\mathrm{OH}, \mathrm{R}_{6}=\mathrm{OC}_{2} \mathrm{H}_{5}$<smiles>[R2]OC(=O)c1ccc(O)c([R])c1</smiles>

(129) $\mathrm{R}_{1}=\mathrm{H}, \mathrm{R}_{2}=\mathrm{CH}_{3}$

(130) $\mathrm{R}_{1}=\mathrm{OCH}_{3}, \mathrm{R}_{2}=\mathrm{H}$

(134) $\mathrm{R}_{1}=\mathrm{OH}, \mathrm{R}_{2}=\mathrm{CH}_{3}$<smiles>O=C1CCC(O)c2cccc(O)c21</smiles>

(133)
HO<smiles>CCCOc1c(O)cc2c(=O)oc3c(O)c(O)cc4c(=O)oc1c2c34</smiles>

(120) $\mathrm{R}=\mathrm{CH}_{3}$

(121) $\mathrm{R}=\mathrm{H}$<smiles>[R]OC(=O)/C=C/c1ccc([R])c([R])c1</smiles>

(127) $\mathrm{R}_{1}=\mathrm{OH}, \mathrm{R}_{2}=\mathrm{CH}_{2}\left(\mathrm{CH}_{2}\right)_{16} \mathrm{CH}_{3}$

(128) $\mathrm{R}_{1}=\mathrm{OCH}_{3}, \mathrm{R}_{2}=\mathrm{CH}_{2}\left(\mathrm{CH}_{2}\right)_{24} \mathrm{CH}_{3}$<smiles>COC1=CC(C(=O)OC2OC(CO)[C@H](O)[C@H](O)[C@H]2O)=CC(OC)C1O</smiles>

(132)

Figure 9: The structures of 19 phenols (117-135).

gastric cancer cell SGC7901/ADR to the chemotherapy drugs paclitaxel and adriamycin, which was manifested in two aspects: it increased the growth inhibitory effects of paclitaxel and adriamycin to SGC7901/ADR gastric cancer cells and significantly reduced the IC50s of paclitaxel and adriamycin to SGC7901/ADR cells, in a concentration-dependent manner [73].

3.4. Liver and Breast Cancers. ELB has also exhibited inhibitory effects on the growth of liver and breast cancer cells. Zhang et al. reported that an ethylacetate extract of ELB could inhibit the growth of ZR-75-30 breast cancer cells, where the inhibition rate increased with an increase in drug concentration [74]. Gao et al. [75] found that an extract of ELB inhibited the proliferation of human hepatoma HepG2 cells in a time- and concentration-dependent manner, which may have been related to mitochondrial pathways or cellular apoptosis pathways. Wang et al. [32] isolated four compounds (compounds 20 and 25-27) which exhibited strong inhibitory activities against HepG2 human hepatocellular carcinoma cells. Liu et al. [35] isolated five compounds (compounds 28-31,53) and evaluated their in vitro antiproliferative activities against MCF-7 breast cancer cells. The results showed that compound $\mathbf{5 3}$ exhibited marked activity, with an IC50 value of $18.6 \mu \mathrm{M}$, and compounds 28-31 showed moderate cytotoxic activities, with IC50 values ranging from 32.1 to $57.1 \mu \mathrm{M}$. 
<smiles>CC(=O)OC[C@H](Cc1ccccc1)NC(=O)[C@H](Cc1ccccc1)NC(=O)c1ccccc1</smiles>

(136)<smiles>NC(=O)c1c[nH]c2ccccc12</smiles>

(137)<smiles>CC(=O)NCCc1c[nH]c2ccccc12</smiles>

(138)<smiles>CC(=O)NCCCc1ccc(C)cc1</smiles>

(139)<smiles>CN1CC(O)C1O</smiles>

(140)

Figure 10: The structures of 5 alkaloids (136-140).<smiles>CC1=CC(=O)CC1(C)C</smiles>

(145)<smiles>O=CCc1ccccc1</smiles>

(146)

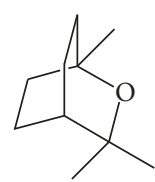

(148)<smiles>CC(C)(C)O</smiles>

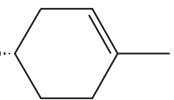

(147)

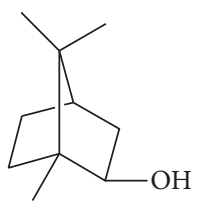

(149)
FIGURE 11: The structures of 5 volatile oils (145-149).
(150)

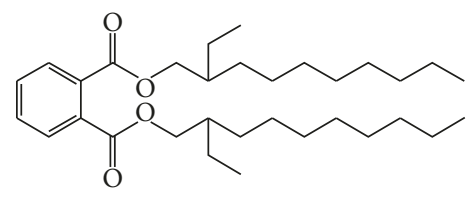<smiles>OC[C@H]1O[C@@H](O[C@@]2(CO)O[C@H](CO)[C@@H](O)[C@H]2O)[C@H](O)[C@H](O)[C@@H]1O</smiles>

(151)
FIGURE 12: The structures of 2 compounds (150-151).

\section{Discussion and Conclusion}

ELB is a traditional Chinese medicine possessing the functions of expectoration, cough relief, asthma relief, detoxification, and itching relief. Modern pharmacological studies have shown that ELB exhibits a variety of activities, such as antitumor, antibacterial, and antioxidant. In particular, its anticancer activities have been paid much attention in recent years. The present review summarizes the chemical constituents and the antitumor activities. The results showed that more than 151 components have been identified from extracts of ELB, including 73 terpenoids, 28 flavonoids, 8 phenylpropanoids, 7 steroids, 19 phenols, and 5 alkaloids. ELB has been shown to exhibit significant inhibitory effects on lung, cervical, gastric, breast, and liver cancers. However, there are limitations associated with most of these studies: (1) most of the studies reported to date have focused exclusively on the use of ELB fractions, as well as the use of its crude extracts; in contrast, there have been very few reports pertaining to the use of single compounds isolated from ELB; (2) most of the studies concerning the anticancer activities and mechanisms have been conducted using in vitro cellular systems, which could explain how nonspecific cell cycle arrest and apoptosis were induced. However, very few reports have focused on specific molecular targets or enzymatic pathways; and (3) to our knowledge, there exist no studies concerned with the anticancer mechanisms of single compounds isolated from ELB. Therefore, systematic research should be carried out to isolate the monomer components and screen the anticancer activity of these components, in order to confirm the anticancer active ingredients and further study on the anticancer activities and mechanisms of these ingredients, which will be useful in discovering new active anticancer single compounds and new anticancer drug resources.

\section{Conflicts of Interest}

The authors declare that there are no conflicts of interest regarding the publication of this paper.

\section{Acknowledgments}

This work was financially supported by the Special Subsidy for Public Health Services of Traditional Chinese Medicine in 2018 "National Survey of Traditional Chinese Medicine Resources" (CS [2018]; no. 43) and the Science and Technology Development Plan Project for Traditional Chinese Medicine of Shandong Province (nos. 2019-0977 and 2019-0978). 


\section{References}

[1] P. P. Hsu and D. M. Sabatini, "Cancer cell metabolism: Warburg and beyond," Cell, vol. 134, no. 5, pp. 703-707, 2008.

[2] A. Jemal, F. Bray, M. M. Center, J. Ferlay, E. Ward, and D. Forman, "Global cancer statistics," CA: A Cancer Journal for Clinicians, vol. 61, no. 2, pp. 69-90, 2011.

[3] Y. Itatani, K. Kawada, and Y. Sakai, "Treatment of elderly patients with colorectal cancer," BioMed Research International, vol. 2018, Article ID 2176056, 8 pages, 2018.

[4] O. Le Saux and C. Falandry, "Toxicity of cancer therapies in older patients," Current Oncology Reports, vol. 20, no. 8, p. 64, 2018.

[5] B. Zhang, C. Fang, D. Deng, and L. Xia, "Research progress on common adverse events caused by targeted therapy for colorectal cancer," Oncology Letters, vol. 16, no. 1, pp. 27-33, 2018.

[6] G. T. Carter, "Natural products and pharma 2011: strategic changes spur new opportunities," Natural Product Reports, vol. 28, no. 11, pp. 1783-1789, 2011.

[7] J. M. Pezzuto, "Plant-derived anticancer agents," Biochemical Pharmacology, vol. 53, no. 2, pp. 121-133, 1997.

[8] R. El-Galley, T. E. Keane, and C. Sun, "Camptothecin analogues and vinblastine in the treatment of renal cell carcinoma: an in vivo study using a human orthotopic renal cancer xenograft," Urologic Oncology: Seminars and Original Investigations, vol. 21, pp. 49-57, 2003.

[9] M. K. Chen and F. Xue, "Paclitaxel-based regimen for the treatment of 24 cases of advanced malignant tumors," Journal of Oncology, vol. 10, no. 5, pp. 373-374, 2004.

[10] X. L. Li, Q. L. Ren, Y. W. Yang et al., "Study on the extraction, purification and analysis methods of antitumor drug vinblastine," China Pharmaceutical Industry Journal, vol. 25, no. 4, pp. 247-251, 2004.

[11] Y. X. Ye, "Micronuclear effect of anticancer drug vincristine," Journal of Railway Teachers Academy, vol. 18, no. 1, pp. 53-55, 2001.

[12] Y. Cai, Q. Luo, M. Sun, and H. Corke, "Antioxidant activity and phenolic compounds of 112 traditional Chinese medicinal plants associated with anticancer," Life Sciences, vol. 74, no. 17, pp. 2157-2184, 2004.

[13] G. M. Cragg, P. G. Grothaus, and D. J. Newman, "Impact of natural products on developing new anti-cancer agents," Chemical Reviews, vol. 109, no. 7, pp. 3012-3043, 2009.

[14] S. Fulda, "Modulation of apoptosis by natural products for cancer therapy," Planta Medica, vol. 76, no. 11, pp. 1075-1079, 2010.

[15] S. Banerjee, Z. Wang, M. Mohammad, F. H. Sarkar, and R. M. Mohammad, "Efficacy of selected natural products as therapeutic agents against cancer," Journal of Natural Products, vol. 71, no. 3, pp. 492-496, 2008.

[16] A.-T. Hauser and M. Jung, "Targeting epigenetic mechanisms: potential of natural products in cancer chemoprevention," Planta Medica, vol. 74, no. 13, pp. 1593-1601, 2008.

[17] R. G. Mehta, G. Murillo, R. Naithani, and X. Peng, "Cancer chemoprevention by natural products: how far have we come?," Pharmaceutical Research, vol. 27, no. 6, pp. 950-961, 2010.

[18] Y. Q. Yan, "Thoughts and methods of modernization of traditional Chinese medicine," Chemical Industry Press, vol. 1, no. 20 , p. $864,2006$.

[19] Chinese Botany Editorial Board and Chinese Academy of Sciences, Flora of China, Science Press, Beijing, China, 1993.
[20] Jiangsu New Medical College, Dictionary of Traditional Chinese Medicine: Volume II, Shanghai Science and Technology Press, Shanghai, China, 1977.

[21] X. W. Chen, "Preliminary study on antibacterial activity of Euphorbia lunulata Bunge.," Ningxia Journal of Agriculture and Forestry Science and technology, vol. 5, pp. 19-20, 2005.

[22] R. Li, Y. Wang, H. X. Wu et al., "Isolation, identification and activity determination of antioxidant active components in Euphorbia lunulata Bunge.," Journal of Shenyang Pharmaceutical University, vol. 28, no. 1, pp. 25-29, 2011.

[23] B. H. Yan, J. Z. Liu, S. M. Dong et al., "Clinical observation of treatment of cervical ulcer lymphatic tuberculosis with Euphorbia lunulata Bunge.," Chinese Pharmacology and Clinical Medicine, vol. 2, pp. 170-171, 2015.

[24] K. L. Liu, “Zhang minghe's experience in treating rheumatic immune diseases with Euphorbia lunulata Bunge. and Ranunculus ternatus thunb," Hunan Journal of Traditional Chinese Medicine, vol. 7, pp. 27-28, 2014.

[25] S. R. Teng and X. L. Li, "Therapeutic effect of 10 cases of psoriasis treated with Euphorbia lunulata Bunge.," Chinese Community Physician, vol. 21, no. 21, p. 34, 2005.

[26] J. Chi, S. P. Wang, and H. P. Yu, "20 cases of caterpillar relief of lung cancer symptoms," Chinese Folk Therapy, vol. 11, no. 8 , p. 51, 2003.

[27] Y. Liu and J. S. Wu, "Clinical observation of anti-disease III combined with chemotherapy in the treatment of advanced gastric cancer," Journal of Shandong University of Traditional Chinese Medicine, vol. 26, no. 6, p. 444, 2002.

[28] Z.-Q. Lu, M. Yang, J.-Q. Zhang et al., "Ingenane diterpenoids from Euphorbia esula," Phytochemistry, vol. 69, no. 3, pp. 812-819, 2008.

[29] Y.-B. Wang, P. Ji, H.-B. Wang, and G.-W. Qin, "Diterpenoids from Euphorbia esula," Chinese Journal of Natural Medicines, vol. 8, no. 2, pp. 94-96, 2010.

[30] S. Kupchan, I. Uchida, A. Branfman, R. Dailey, and B. Fei, "Antileukemic principles isolated from euphorbiaceae plants," Science, vol. 191, no. 4227, pp. 571-572, 1976.

[31] A. Marston and E. Hecker, "On the active principles of the Euphorbiaceae," Planta Medica, vol. 47, no. 3, pp. 141-147, 1983.

[32] J. Wang, X. F. Li, P. Xie et al., "Study on antitumor active components of Euphorbia lunulata," Chinese Herbal Medicines, vol. 43, no. 10, pp. 1891-1895, 2012.

[33] J. Li, S. Wu, M. Zhao et al., "A new jatrophane-type diterpene in Euphorbia lunulata Bunge.," Chinese Herbal Medicines, vol. 46, no. 14, pp. 2045-2047, 2015.

[34] M. Zhao, S. Wu, J. Li et al., "Study on chemical constituents of Euphorbia lunulata Bunge.," China Journal of Chinese Materia Medica, vol. 39, no. 12, pp. 2289-2294, 2014.

[35] C. Liu, Z.-X. Liao, S.-J. Liu, Y.-B. Qu, and H.-S. Wang, “Two new diterpene derivatives from Euphorbia lunulata Bge and their anti-proliferative activities," Fitoterapia, vol. 96, pp. 33-38, 2014.

[36] A. Vasas, E. Sulyok, D. Rédei et al., "Jatrophane diterpenes from Euphorbia esulaas antiproliferative agents and potent chemosensitizers to overcome multidrug resistance," Journal of Natural Products, vol. 74, no. 6, pp. 1453-1461, 2011.

[37] J. Hohmann, A. Vasas, G. Günther et al., "Macrocyclic diterpene polyesters of the jatrophane type from Euphorbia esula," Journal of Natural Products, vol. 60, no. 4, pp. 331-335, 1997.

[38] G. Günther, J. Hohmann, A. Vasas, I. Máthé, G. Dombi, and G. Jerkovich, "Jatrophane diterpenoids from Euphorbia esula," Phytochemistry, vol. 47, no. 7, pp. 1309-1313, 1998. 
[39] L. G. Liu, J. C. Meng, S. X. Wu, X. Y. Li, X. C. Zhao, and R. X. Tan, "New macrocyclic diterpenoids from Euphorbia esula," Planta Medica, vol. 68, no. 3, pp. 244-248, 2002.

[40] T. Sekine, M. Kamiya, F. Ikegami, and J.-F. Qi, "Structure of Esulol A, a novel macrocyclic diterpene, from inner Mongolian Euphorbia Esula," Natural Product Letters, vol. 12, no. 3, pp. 237-239, 1998.

[41] W. J. Zhang, L. J. Weng, L. T. Yi et al., "Study on the chemical constituents of Euphorbia lunulata Bunge.," Chinese Herbal Medicines, vol. 47, no. 4, pp. 554-558, 2016.

[42] Y.-B. Wang, H.-B. Wang, P. Ji, J. Guo, H.-Z. Jin, and G.-W. Qin, "Esulatin G, a novel nor-diterpenoid from Euphorbia esula," Chemistry Letters, vol. 38, no. 3, pp. 270-271, 2009.

[43] W. J. Zhang, Study on Chemical Constituents and Antitumor Activity of Euphorbia Lunulata Bge, Huaqiao University, Quanzhou, China, 2016.

[44] G. S. Chai, Study on the Chemical Constituents of the Seeds of Euphorbia Fischeriana Steud. and Euphorbia Esula Linn. Qiqihar University, Qiqihar, China, 2012.

[45] C. Liu, H. Sun, W. T. Wang et al., "Study on chemical constituents of Euphorbia lunulata bge.," Journal of Chinese Medicinal Materials, vol. 3, pp. 514-517, 2015.

[46] Z.-G. Yang, L.-N. Jia, Y. Shen, A. Ohmura, and S. Kitanaka, "Inhibitory effects of constituents from Euphorbia lunulata on differentiation of 3T3-L1 cells and nitric oxide production in RAW264.7 cells," Molecules, vol. 16, no. 10, pp. 8305-8318, 2011.

[47] T. M. Shang, L. Wang, X. T. Liang et al., "Study on the chemical constituents of Euphorbia Lunulata," Journal of Chemistry, vol. 37, no. 2, pp. 119-128, 1979.

[48] T. Nishimura, L.-Y. Wang, K. Kusano, and S. Kitanaka, "Flavonoids that mimic human ligands from the whole plants of Euphorbia lunulata," Chemical \& Pharmaceutical Bulletin, vol. 53, no. 3, pp. 305-308, 2005.

[49] F. Halaweish, S. Kronberg, and J. Rice, "Rodent and ruminant ingestive response to flavonoids in Euphorbia esula," Journal of Chemical Ecology, vol. 29, no. 5, pp. 1073-1082, 2003.

[50] D. X. Wang and X. T. Liang, "Structure and stereochemistry of maoyancaosu," Journal of Pharmaceutical Sciences, vol. 19, no. 4, pp. 261-267, 1984.

[51] R. Li and N. L. Wang, "Study on phenolic acids and lignans in Euphorbia lunulata Bunge.," Nei Menggu Journal of Chinese Traditional Medicine, vol. 31, no. 11, pp. 87-88, 2012.

[52] J. H. Rong, Study on the Antioxidant and Antitumor Effects of Euphorbia Lunulata Bge.Qingdao University of Science and Technology, Qingdao, China, 2014.

[53] X. Wang, H. L. Su, W. M. Li et al., "GC-MS analysis of volatile oil components in Euphorbia lunulata Bunge.," Northwest Pharmaceutical Journal, vol. 4, pp. 353-356, 2016.

[54] J. C. Reed, "Dysregulation of apoptosis in cancer," Journal of Clinical Oncology, vol. 17, no. 9, p. 2941, 1999.

[55] D. E. Fisher, "Apoptosis in cancer therapy: crossing the threshold," Cell, vol. 78, no. 4, pp. 539-542, 1994.

[56] J. M. Brown and L. D. Attardi, "The role of apoptosis in cancer development and treatment response," Nature Reviews Cancer, vol. 5, no. 2, p. 231, 2005.

[57] C. Kim and B. Kim, "Anti-cancer natural products and their bioactive compounds inducing ER stress-mediated apoptosis: a review," Nutrients, vol. 10, no. 8, p. 1021, 2018.

[58] A. Jemal, M. M. Center, C. DeSantis, and E. M. Ward, "Global patterns of cancer incidence and mortality rates and trends," Cancer Epidemiology Biomarkers \& Prevention, vol. 19, no. 8, pp. 1893-1907, 2010.

[59] B. H. Xiao, C. L. Zhang, and X. Wu, "Inhibitory effect of aqueous extract of cat's eye grass on proliferation of Lewis lung carcinoma in mice and its mechanism," China Journal of Chinese Materia Medica, vol. 31, no. 12, pp. 1002-1005, 2006.

[60] C. L. Zhang and B. H. Xiao, "Effects of aqueous extract of Euphorbia lunulata Bunge. on the expression of Caspase-3 in Lewis lung cancer tissues," Journal of Clinical Oncology, vol. 11, no. 12, pp. 901-903, 2006.

[61] S. Jiang, The Inhibition of Lung Cancer Active Ingredient Extracts of Euphorbia Lunulata and its Mechanism, Ocean University of China, Qingdao, China, 2011.

[62] A. H. Wang, Q. X. Pang, M. N. Chen et al., "Effects of Euphorbia lunulata extract on the growth of human lung cancer cells," Journal of Shanxi Medical University, vol. 45, no. 6, pp. 460-464, 2014.

[63] J. M. M. Walboomers, M. V. Jacobs, M. M. Manos et al., "Human papillomavirus is a necessary cause of invasive cervical cancer worldwide," The Journal of Pathology, vol. 189, no. 1, pp. 12-19, 1999.

[64] P. H. Ming, H. B. Chen, T. Cai et al., "Study on apoptosis of HeLa cells induced by extract of Euphorbia lunulata and its mechanism," Shizhen Guo Yi Guo Yao, vol. 24, no. 6, pp. 1318-1321, 2013.

[65] J. J. Tu, L. Wang, Q. Q. Ren et al., "Study on inhibition of proliferation and migration of human cervical cancer cells and induction of apoptosis by Euphorbia lunulata," Journal of Yan'an University (Medical Sciences), vol. 14, no. 4, pp. 1-5, 2016.

[66] M. Bar-Zeev, Y. G. Assaraf, and Y. D. Livney, " $\beta$-casein nanovehicles for oral delivery of chemotherapeutic drug combinations overcoming P-glycoprotein-mediated multidrug resistance in human gastric cancer cells," Oncotarget, vol. 7, no. 17, p. 23322, 2016.

[67] W. Cao, W. Wei, Z. Zhan, Y. Xie, and Q. Xiao, "miR-1284 modulates multidrug resistance of gastric cancer cells by targeting EIF4A1," Oncology Reports, vol. 35, no. 5, pp. 2583-2591, 2016.

[68] H. Chen, J. Shen, E. Choy, F. J. Hornicek, and Z. Duan, "Targeting protein kinases to reverse multidrug resistance in sarcoma," Cancer Treatment Reviews, vol. 43, pp. 8-18, 2016.

[69] K. Moitra, "Overcoming multidrug resistance in cancer stem cells," BioMed Research International, vol. 2015, Article ID 635745, 8 pages, 2015.

[70] Y. Wang, K. Wu, Z. Yang et al., "Multidrug-resistance related long non-coding RNA expression profile analysis of gastric cancer," PLoS One, vol. 10, no. 8, Article ID e0135461, 2015.

[71] X. L. Guo, Z. Y. Fu, Y. Bi et al., "Study on the inhibition of proliferation, migration, invasion and induction of apoptosis in human gastric cancer multidrug resistant cells by Chinese herbal medicine sputum," Journal of Biomedical Engineering, vol. 35, no. 2, pp. 244-251, 2018.

[72] Z. Fu, X. Han, J. Du et al., "Euphorbia lunulata extract acts on multidrug resistant gastric cancer cells to inhibit cell proliferation, migration and invasion, arrest cell cycle progression, and induce apoptosis," Journal of Ethnopharmacology, vol. 212, pp. 8-17, 2018.

[73] W. B. Wang, X. X. Han, Z. Y. Fu et al., "Study on the sensitivity of multidrug resistant gastric cancer cells to chemotherapeutic drugs in sputum sputum," Journal of Yan'an University (Medical Sciences), vol. 15, no. 4, pp. 1-6, 2017.

[74] Y. Zhang, L. Zheng, W. He et al., "Antitumor activity of ethyl acetate in cat's eye grass," World Science and TechnologyModernization of Traditional Chinese Medicine, vol. 19, no. 10, pp. 1729-1736, 2017.

[75] F. Gao, Z. Fu, H. Tian, and Z. He, "The Euphorbia lunulata Bge extract inhibits proliferation of human hepatoma Hep G2 cells and induces apoptosis," Journal of Buon, vol. 18, no. 2, pp. 491-495, 2013. 


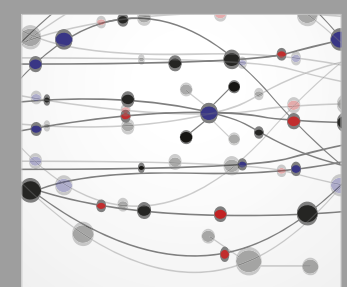

The Scientific World Journal

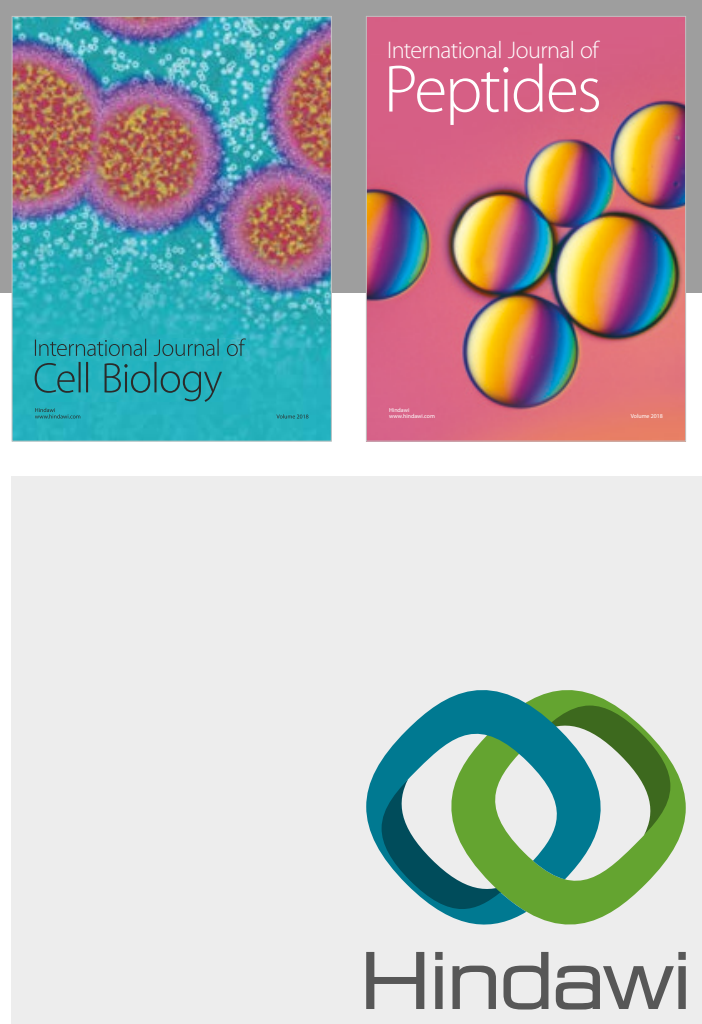

Submit your manuscripts at

www.hindawi.com
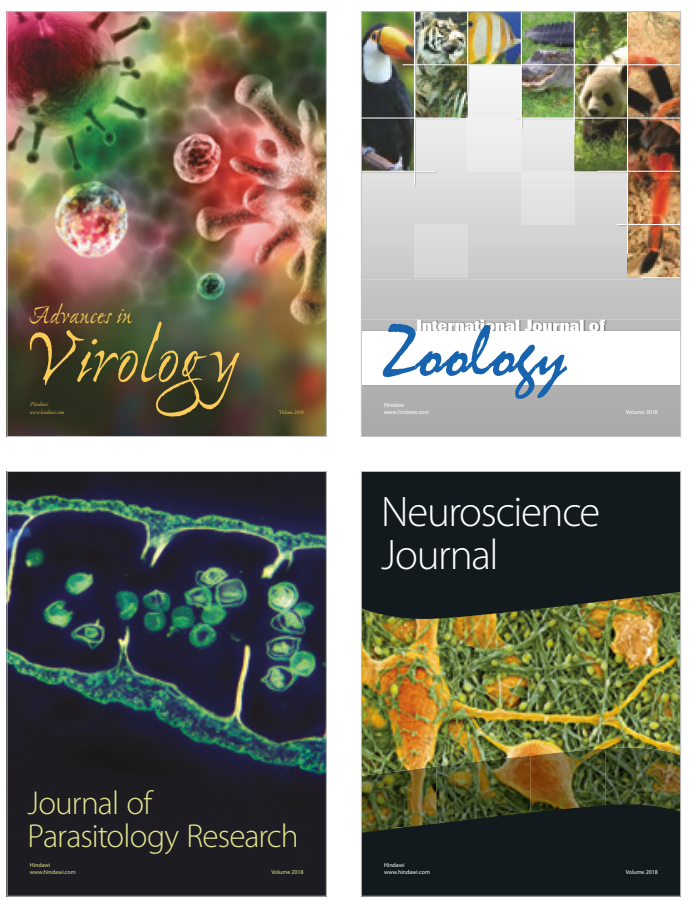
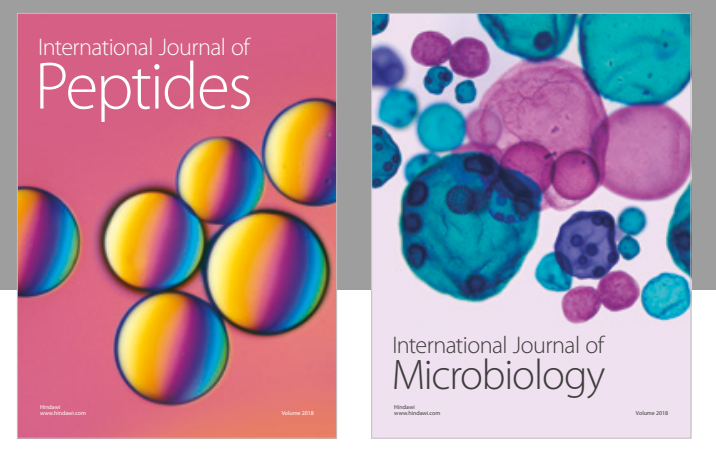

nternational Journal of Microbiology
Journal of
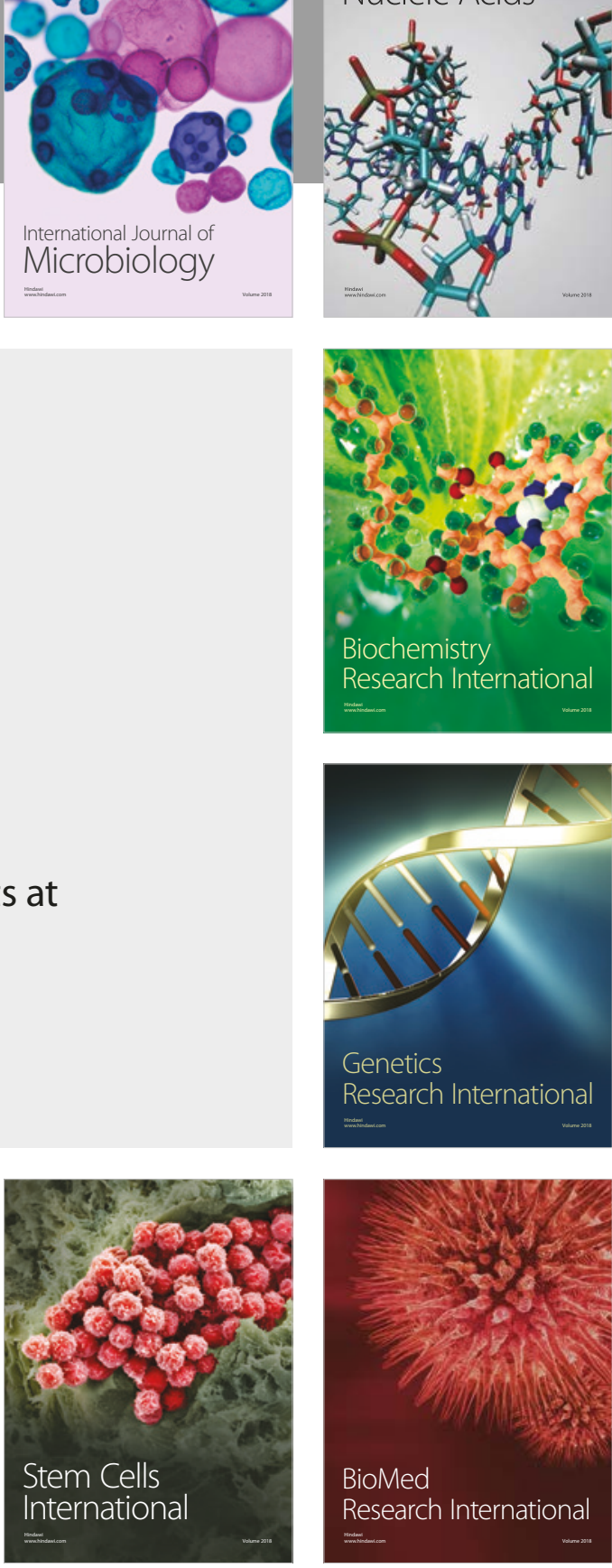
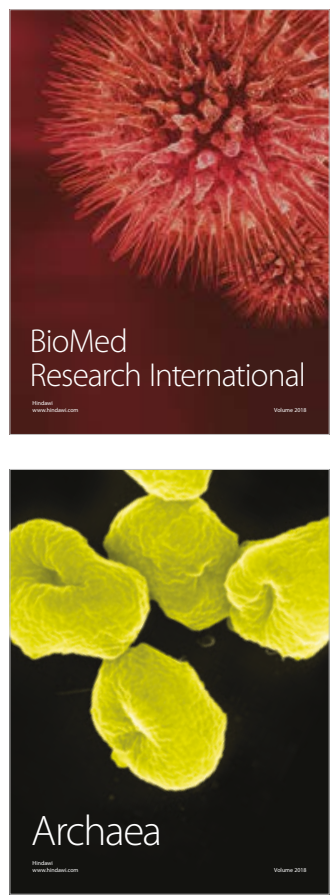